\title{
Error diagnosis in finite communicating systems
}

\author{
Anne RASSE*
}

\begin{abstract}
We present an error diagnosis method for parallel communicating systems with branching temporal specifications. Verification is done by model checking on the finite graph of the executions. We consider errors whose diagnostics are sequences of the graph. We define a minimality criterium for the diagnostics such that a finite number of minimal diagnostics give all the reasons of the error. Diagnostics are produced in a simplified form according to a given abstraction. We define an equivalence on models which preserves the simplified diagnostics.
\end{abstract}

\section{Introduction}

The complexity and the growing importance of parallel and reactive systems [Pnu86] requires the development of validation tools. Validation consists successively in detecting the errors, localizing, then correcting them. Error detection is done by comparing the executions of the program against a reference obtained from the service specifications. It can be performed either by verification, simulation or test. When an error is detected, it is necessary to find out the reasons for the error in order to correct it. We present a diagnosis method for verification by model checking, a method used by systems like MEC [Arn89], EMC [CES83], XESAR [RRSV87] : a state graph of the behaviour of the program is compared to specifications given by formulas of temporal logic. Our diagnostics explain the non-validity of the specifications in terms of sequences of the model. It has been shown that every specification of a system can be expressed in terms of safety and liveness properties [AL88]. Intuitively, a safety property expresses that something bad can never happen, and a liveness property expresses that something good eventually happens. They concern all executions of the program : if they are not satisfied, there exists an execution where something bad happens or something good does not happen. Such an execution is called an explicative sequence.

The diagnosis method presented here has been implemented in the framework of XESAR, a verification tool for communication protocols. The model for the executions of the program is a finite state graph. Specifications are expressed in the branching temporal logic CTL [CES83]. The semantics of CTL is defined by a satisfaction relation $=$ between states of the graph and the formulas. The verification examines if all the states satisfy $f$. If it is not the case, an error diagnostics is generated, which is an explanation of an assertion $s \models \neg f:$ it expresses properties of the graph proving $s \models \neg f$, such as explicative sequences.

\footnotetext{
* LGI IMAG Campus BP53X 38041 Grenoble cedex France; e-mail: \{rasse\}oimag.imag.er
} 
This paper is organized as follows. In section 1 , we give the model of the program, and the language of formulas. Section 2 is devoted to the generation of explanations. An assertion has generally an infinite number of explanations, but we show that every assertion has a finite number of minimal one giving all the reasons explaining it. In section 3, we are interested in simplification of explanations according to a set of visible actions. We define an equivalence relation between models of a formula $f$, the explanational equivalence, which preserves the simplified explanations of $f$. In section 4, we present computation of explanations by the diagnostics tool of XESAR, called CLÉO, which implements our diagnosis method.

\section{Model of programs - Language of specifications}

- Model of programs. Let $S$ be a finite set of states, $s_{0}$ the initial state, Act a set of identifiers associated by the user to actions of the program, $\rightarrow \subset S \times A c t \times S$ a transition relation labelled with elements of $A c t, \mathcal{P}$ a set of propositional variables, and a total labelling function $\Pi: S \rightarrow 2^{\mathcal{P}}$. $\mathcal{P}$ contains a variable sink which labels the states whithout successors. The model of the program is the execution structure $M=\left(S, \rightarrow, s_{0}\right.$, Act, $\left.\mathcal{P}, \Pi\right)$. We write : $s_{1} \stackrel{\alpha}{\rightarrow} s_{2}$ iff $\left(s_{1}, \alpha, s_{2}\right) \in \rightarrow$ and $s_{1} \stackrel{\sigma}{\rightarrow} s_{n}$ with $\sigma=\alpha_{1} \ldots \alpha_{n-1}$ iff $\exists s_{2}, \ldots s_{n-1} \mid \forall i \leq n-1 . s_{i} \stackrel{\alpha_{i}}{\rightarrow}$ $s_{i+1}$. If $w \in S^{*}, w(i)$ is the i-th element of $w$. An execution sequence is a non empty sequence $w$ of states s. t. $\forall i . w(i+1) \rightarrow w(i)$. If $w$ is finite, last $(w)$ is its last element. $w$ is maximal if $w$ is infinite or last $(w)$ is a sink state. $E x(s)$ is the set of execution sequences of state $s$ and $M x(s)$ the set of maximal ones. $S^{c} s$ the set of the infinite cyclic sequences : $S^{c}=\left\{w_{1}\left(w_{2}\right)^{\omega} \mid w_{1}, w_{2} \in S^{*}\right\}$.

- Specification language : CTL without next time operator [EC82].

- Syntax. The formulas of CTL are defined by the abstract grammar :

$$
f::=T|p \in \mathcal{P}| \neg f|f \vee f| E(f \mathcal{U} f) \mid A(f \mathcal{U} f)
$$

- Semantics. The semantics is defined by a satisfaction relation between states and formulas. We write $M, s \vDash f$, or $s \vDash f$ when $M$ is clear from the context, if the state $s$ satisfies $f$. The relation $\models$ is defined inductively over the structure of the formulas as follows :

$$
\begin{aligned}
& \forall s \in S, s \neq T \\
& s \vDash p \Leftrightarrow p \in \Pi(s) \\
& s \vDash \neg f \Leftrightarrow s \not \neq f \\
& s \vDash f_{1} \vee f_{2} \Leftrightarrow\left(s \neq f_{1} \text { or } s \neq f_{2}\right) \\
& s=E\left(f_{1} \mathcal{U} f_{2}\right) \Leftrightarrow\left(\exists w \in M x(s), \exists k \geq 1 \mid\left(\forall i<k . w(i) \models f_{1}\right) \text { and } w(k) \models f_{2}\right) \\
& s \vDash A\left(f_{1} \mathcal{U} f_{2}\right) \Leftrightarrow\left(\forall w \in M x(s), \exists k \geq 1 \mid\left(\forall i<k . w(i) \vDash f_{1}\right) \text { and } w(k) \models f_{2}\right)
\end{aligned}
$$

For convenience, in the following, we use the abbreviations :

$$
\begin{gathered}
f_{1} \wedge f_{2}=\neg\left(\neg f_{1} \vee \neg f_{2}\right) \quad f_{1} \Rightarrow f_{2}=\neg f_{1} \vee f_{2} \quad \operatorname{pot}\left[f_{1}\right] f_{2}=E\left(f_{1} \mathcal{U} f_{2}\right) \\
\text { inev }\left[f_{1}\right] f_{2}=A\left(f_{1} \mathcal{U} f_{2}\right) \quad \text { al }\left[f_{1}\right] f_{2}=\neg \operatorname{pot}\left[f_{1}\right] \neg f_{2} \quad \text { some }\left[f_{1}\right] f_{2}=\neg \operatorname{inev}\left[f_{1}\right] \neg f_{2}
\end{gathered}
$$

and we write op $f$ instead of $o p[T] f$ for $o p=p o t$, some, al or inev.

The program satisfies the formula $f$ iff $\forall s \in S, s \neq f$. Thus, the specifications are not valid if there exists a state $s$ and a formula $f$ in the specifications such that $s \vDash \neg f$. The aim of our method is to explain why an assertion $s \models \neg f$ is true. 


\section{The method for generation of explanations}

\subsection{Principle}

We produce explanations for the formulas whose satisfaction can be shown by giving a single execution sequence. This allows to explain the non satisfaction of formulas constructed from the propositional variables, their negations, operators $\wedge, \vee$, al and inev. Most of the usefull specifications can be expressed by such formulas. In fact, a safety property meaning that something bad can never happen, is generally expressed by a formula like al $\neg$ "bad". A liveness property meaning that someting good does eventually happen is generally expressed by formulas like inev "good" or al inev "good". The negation of these formulas contains only the modalities pot and some in the explication of which we are especially interested.

The method is extended to the other formulas of CTL. A propositional variable $p$ or its negation does not require any explanation : the assertions $s \models p$ (or $s \models \neg p$ ) are their own explanation. Formulas like al $\left[f_{1}\right] f_{2}$ or inev $\left[f_{1}\right] f_{2}$ cannot be explained in terms of execution sequences, because they express properties of all executions sequences : to explain $s \vDash$ $a l\left[f_{1}\right] f_{2}$ or $s \vDash \operatorname{inev}\left[f_{1}\right] f_{2}$, we have to give all execution sequences from $s$, which would be an information too large to be useful. So, $s \vDash a l\left[f_{1}\right] f_{2}$ and $s \vDash i n e v\left[f_{1}\right] f_{2}$ are their own explanation. We call basic formulas the formulas which we do not explain : the propositional variables, their negations, and the formulas equivalent to a formula al $\left[f_{1}\right] f_{2}$ or inev $\left[f_{1}\right] f_{2}$.

The first step of the generation of an explanation of $s \neq f$ is to write $f$ in a canonical form $\bigvee_{i} \bigwedge_{j}\left(f_{i, j}\right)$, where all $f_{i, j}$ are basic formulas, or of the form op $\left[g_{1}\right] g_{2}$ (op=pot or some), $g_{1}$ and $g_{2}$ being in canonical form. Then, the generation of explanations is based on a stepwise explanation of the sub-formulas : $s$ satisfies $f$ because some sub-formulas of $f$ are satisfied in some states reachable from $f$. Explanations are terms built from assertions of the form $s_{i} \vDash f_{i}$. An explanation of $s \models f$ is obtained by using a rewrite system : a rule rewrites $s \models f$ into a term whose the assertions concern sub-formulas of $f$, which have to be explained at their turn. The generation of an explanation terminates when the formulas appearing in the assertions are basic formulas. Consider for example the assertion $s \vDash p$ pot $\left[f_{1}\right] f_{2}$ :

$s=\operatorname{pot}\left[f_{1}\right] f_{2} \quad$ because there exists an execution sequence $w$ from $s$ in which a

state satisfies $f_{2}$ and each previous state on $w$ satisfies $f_{1}$.

The explanation of this assertion (and also of $s \models s o m e\left[f_{1}\right] f_{2}$ ) requires to exhibit a particular sequence explaining this assertion. We study these explicative sequences $(e s)$ in the next section.

\subsection{Explicative sequences}

If $f=\operatorname{pot}\left[f_{1}\right] f_{2}$ or $f=\operatorname{some}\left[f_{1}\right] f_{2}$, we denote by $W(s \models f)$ the set of es of $s \neq f$. We show that there exists a partial order over $W(s \models f)$, compatible with the natural order induced by the notion of sub-sequences, such that there is a finite number of minimal elements, and 
that every es has a lower bound which is a minimal element. Consequently, if the sequence $w$ explains $s \models f$, there exists a minimal es comparable with $w$ which explains also $s \models f$.

\subsubsection{Explicative sequences of $s \models \operatorname{pot}\left[f_{1}\right] f_{2}$}

- Definition. $s \models \operatorname{pot}\left[f_{1}\right] f_{2}$ is the case iff $\exists s_{1} \ldots s_{i} \ldots \in M x(s)$ and $\exists k>0$ s.t. $s_{1}=s$ and $\left(\forall i<k . s_{i} \models f_{1}\right)$ and $s_{k} \models f_{2}$. Let $a$ be the assertion $s \models p o t\left[f_{1}\right] f_{2}$, then

$$
W(a)=\left\{s_{1} \ldots s_{k} \in E x(s) \mid\left(\forall i<k, s_{i} \vDash f_{1}\right) \text { and } s_{k} \vDash f_{2}\right\}
$$

- Properties. If $w=s_{1} \ldots s_{k} \in W(a)$, some shorter es of $a$ may be extracted from $w$ :

- by prefix : if $\exists i<k \mid s_{i} \vDash f_{2}$, then $s_{1} \ldots s_{i}$ is also an es of $a$.

- by pruning away a cycle $:$ if $\exists i<j \mid s_{i}=s_{j}$, then $s_{1} \ldots s_{i} s_{j+1} \ldots s_{k}$ is also an es of $a$.

This leads us to define an order relation $\leq_{f i}$ on finite sequences : $\leq_{f i}$ is the transitive closure of $\leq_{f i}^{0}$, s.t. $w_{1} \leq_{f i}^{0} w_{2}$ iff $w_{1}$ is a prefix of $w_{2}$, or $w_{1}$ is obtained from $w_{2}$ by pruning a cycle away. Let $W^{0}(a)$ be the subset of $W(a)$ whose elements have no cycle, and such that none of their strict prefixes belongs to $W(a)$. The following properties are straighforward :

Proposition 1 If $a=s \vDash \operatorname{pot}\left[f_{1}\right] f_{2}: W^{0}(a)$ is the finite set of elements of $W(a)$ minimal for $\leq_{f i}$. Every element of $W(a)$ has a minimal lower bound in $W^{0}(a)$.

\subsubsection{Explicative sequences of $s=\operatorname{some}\left[f_{1}\right] f_{2}$}

- Definition. If $s \models \operatorname{some}\left[f_{1}\right] f_{2}$, i.e. $s \not \nvdash$ inev $\left[f_{1}\right] \neg f_{2}$, there exists an execution sequence from $s$ which cannot reach $\neg f_{2}$ through states always satisfying $f_{1}$. In other words there exists, either : an execution sequence having an occurence of a state satisfying $\neg f_{1}$ before reaching a state satisfying $\neg f_{2}$, or an execution sequence ending without having an occurence of a state satisfying $\neg f_{2}$, or an infinite execution sequence without an occurence of a state satisfying $\neg f_{2}$. The graph of the program being finite, the existence of an infinite sequence is related to the existence of a cycle in this graph. Let $a$ be the assertion $s \neq s o m e\left[f_{1}\right] f_{2} . W(a)$ is the union of a set of finite sequences : $W_{f_{i}}(a)=\left\{s_{1} \ldots s_{k} \in E x(s) \mid\left(\forall i<k . s_{i} \mid=\neg f_{1} \wedge f_{2}\right)\right.$ and $\left(s_{k} \vDash \neg f_{1} \wedge f_{2}\right.$ or $s_{k} \vDash f_{1} \wedge f_{2} \wedge$ sink) $\}$ and a set of cyclic sequences : $W_{\text {in }}(a)=$ $\left\{s_{1} \ldots s_{k}\left(s_{k+1} \ldots s_{n}\right)^{\omega} \in E x(s) \mid s_{k}=s_{n}\right.$ and $\left.\left(\forall i . s_{i} \vDash f_{1} \wedge f_{2}\right)\right\}$

- Properties of $W_{f i}\left(s \models\right.$ some $\left.\left[f_{1}\right] f_{2}\right)$. In the same way as for pot $\left[f_{1}\right] f_{2}$, every es obtained from a finite explicative sequence of $a$ by pruning away a cycle is an es of $a$ (notice that it is never the case for their strict prefixes) Let $W_{f i}^{0}(a)$ contain the elements of $W_{f i}(a)$ whithout cycles. This set has similar properties as $W^{0}\left(s \models p o t\left[f_{1}\right] f_{2}\right)$ :

Proposition 2 If $a=s \models \operatorname{some}\left[f_{1}\right] f_{2}: W_{f i}^{0}(a)$ is the finite set of elements of $W_{f i}(a)$ minimal for $\leq_{f i}$. Every element of $W_{f i}(a)$ has a minimal lower bound in $W_{f i}^{0}(a)$.

- Properties of $W_{i n}\left(s=s o m e\left[f_{1}\right] f_{2}\right)$. We define an order relation on cyclic sequences such that the set of minimal elements of $W_{i n}(a)$ has similar properties as the minimal finites es of $s \vDash \operatorname{pot}\left[f_{1}\right] f_{2}$ or $s \models \operatorname{some}\left[f_{1}\right] f_{2}$. The minimal cyclic es of a must explain this assertion with a minimal information : it should prove the existence of an infinite execution of the 
program whith only one elementary cycle of the graph. Then, the order on cyclic sequences to be considered should take into account the number of elementary cycles covered by the sequences. To compare cyclic sequences, we define a canonical form for them :

Definition $1 \forall w \in S^{c}$, there exists a unique pair of finite sequences $\left(w_{1}, w_{2}\right)$ s. $t$. $w_{2}$ is the first shortest period of $w$ satisfying : $w=w_{1}\left(w_{2}\right)^{\omega}$ and last $\left(w_{1}\right)=\operatorname{last}\left(w_{2}\right) \cdot w_{1}\left(w_{2}\right)^{\omega}$ is the canonical form of $w$.

From now on, we suppose always a cyclic sequence to be given in canonical form. Let $n c\left(w_{1}\left(w_{2}\right)^{\omega}\right)$ be the number of elementary cycles covered by the finite sequence $w_{1} w_{2}$ :

$$
n c\left(w_{1}\left(w_{2}\right)^{\omega}\right)=\operatorname{Card}\left(\left\{(i, j) \mid 1 \leq i<j \leq n \text { and } w_{3}(i)=w_{3}(j)\right\}\right) \text {, where } w_{3}=w_{1} w_{2}
$$

We want to introduce an order relation $\leq_{\text {in }}$ on cyclic sequences such that : $w \leq \leq_{\text {in }} w^{\prime} \Rightarrow$ $n c(w) \leq n c\left(w^{\prime}\right)$. There is a simple way to define such an order, in terms of the relation $\leq_{f i}$ :

$$
\forall w_{1}\left(w_{2}\right)^{\omega}, w_{1}^{\prime}\left(w_{2}^{\prime}\right)^{\omega} \in S^{c}, w_{1}^{\prime}\left(w_{2}^{\prime}\right)^{\omega} \leq_{i n} w_{1}\left(w_{2}\right)^{\omega} \Leftrightarrow w_{1}^{\prime} w_{2}^{\prime} \leq_{f i} w_{1} w_{2}
$$

Indeed, if $W_{i n}^{0}(a)=\left\{w \in W_{i n}(a) \mid n c(w)=1\right\}$, we have the following properties :

Proposition 3 If $a=s \vDash$ some $\left[f_{1}\right] f_{2}: W_{i n}^{0}(a)$ is the finite set of elements of $W_{\text {in }}(a)$ minimal for $\leq_{i n}$. Every element of $W_{\text {in }}(a)$ has a minimal lower bound in $W_{i n}^{0}(a)$.

\subsection{Building the explanations}

The set of explanations is the least set defined by the set of rules described by the following table. The rule corresponding to a row must be understood as "if condition is true then

\begin{tabular}{|c|c|c|c|}
\hline condition & explanation & assertion & name \\
\hline$s \models f$ & $s \models f$ & $s \models f$ & $(I)$ \\
\hline$s \models f_{i}$ & $s \mid=f_{i}$ & $s \equiv f_{1} \vee \ldots \vee f_{n}$ & $(D)$ \\
\hline$s=f_{1} \wedge \ldots \wedge f_{n}$ & $\left(s \mid=f_{1} \& \ldots \& s \models f_{n}\right)$ & $s \models f_{1} \wedge \ldots \wedge f_{n}$ & $(C)$ \\
\hline$w \in W\left(s=p o t\left[f_{1}\right] f_{2}\right)$ & $\operatorname{pot}\left[f_{1}\right] f_{2}: e\left(w, p o t\left[f_{1}\right] f_{2}\right)$ & $s \models \operatorname{pot}\left[f_{1}\right] f_{2}$ & $(P)$ \\
\hline$w \in W\left(s \mid=\operatorname{some}\left[f_{1}\right] f_{2}\right)$ & some $\left[f_{1}\right] f_{2}: e\left(w\right.$, some $\left.\left[f_{1}\right] f_{2}\right)$ & $s \mid=\operatorname{some}\left[f_{1}\right] f_{2}$ & $(S)$ \\
\hline $\begin{array}{l}x \text { is an expl. of } s \models f \\
x \text { contains } s^{\prime} \models f^{\prime} \\
x^{\prime} \text { is an expl. of } s^{\prime} \models f^{\prime}\end{array}$ & $x\left[x^{\prime} / s^{\prime}=f^{\prime}\right]$ & $s \models f$ & $(X)$ \\
\hline
\end{tabular}
explanation is an explanation of the assertion assertion".

where $e(w, f)$ is defined by :

$$
\begin{aligned}
& e\left(s_{1} \ldots s_{n}, \operatorname{pot}\left[f_{1}\right] f_{2}\right)=s_{1} \vDash f_{1} \rightarrow \ldots s_{n-1} \vDash f_{1} \rightarrow s_{n} \vDash f_{2} \\
& e\left(s_{1} \ldots s_{n}, \text { some }\left[f_{1}\right] f_{2}\right)=\left\{\begin{array}{c}
s_{1} \vDash f_{1} \wedge f_{2} \ldots s_{n-1} \vDash f_{1} \wedge f_{2} \rightarrow s_{n} \vDash \neg f_{1} \wedge f_{2} \text { if } s_{n} \not f_{1} \\
s_{1} \vDash f_{1} \wedge f_{2} \rightarrow \ldots s_{n-1} \vDash f_{1} \wedge f_{2} \rightarrow s_{n} \vDash f_{1} \wedge f_{2} \wedge \operatorname{sink} \\
\text { if } s_{n} \vDash f_{1}
\end{array}\right. \\
& e\left(s_{1} \ldots s_{k}\left(s_{k+1} \ldots s_{n}\right)^{\omega}, \text { some }\left[f_{1}\right] f_{2}\right)=s_{1} \vDash f_{1} \wedge f_{2} \rightarrow \ldots s_{k} \vDash f_{1} \wedge f_{2}(\rightarrow \\
& \left.\vDash f_{1} \wedge f_{2} \rightarrow \ldots \rightarrow s_{n} \vDash f_{1} \wedge f_{2}\right)^{\omega}
\end{aligned}
$$

Complete explanations contain only assertions $s \models f$ where $f$ is a basic formula. $X(s \models f)$ denotes the set of explanations of $s \models f$. 
Remark : propositional variables in XESAR. When using CTL to express properties of programs, the propositional variables are interpreted as basic predicates which express properties of the states. In the following, we consider the generation of explanations in the case of XESAR. The set $\mathcal{P}$ includes predicates of the form enable $(\alpha)$ and after $(\alpha)$; where $\alpha \in$ Act. For these predicates, the function $\Pi$ is such that : (enable $(\alpha) \in \Pi(s)$ iff $\left.\exists s^{\prime} \mid s \stackrel{\alpha}{\rightarrow} s^{\prime}\right)$ and $\left(\operatorname{after}(\alpha) \in \Pi(s)\right.$ iff $\exists s^{\prime} \mid s^{\prime} \stackrel{\alpha}{\rightarrow} s$ and $\left.\forall s^{\prime} . s^{\prime} \rightarrow s \Rightarrow s^{\prime} \stackrel{\alpha}{\rightarrow} s\right)$.

Example. Consider the specification for the program on figure 1 : "every transmission $(t m)$ is inevitably followed by a reception( $(r c)$ ", expressed by the formula $f=\operatorname{al}(\operatorname{after}(\operatorname{tm}) \Rightarrow$ inev after $(r c)))$. We build an explanation of $s_{0} \vDash \neg f=\operatorname{pot}(\operatorname{after}(\operatorname{tm}) \wedge$ some $\neg$ after $(r c))$ :

$$
\begin{aligned}
& \text { (30) } \\
& s_{0} \models \neg f \stackrel{(P)}{\mapsto} \neg f:\left(s_{0} \vDash T \rightarrow s_{1} \models \operatorname{after}(\mathrm{em}) \wedge \text { some } \neg a f t e r(r \mathrm{c})\right) \\
& s_{1} \models \operatorname{after}(e m) \wedge \operatorname{some} \neg \operatorname{after}(r c) \stackrel{(C)}{\mapsto}\left(s_{1} \models \operatorname{after}(\mathrm{em}) \& s_{1} \vDash \operatorname{some} \neg a f t e r(r c)\right) \\
& s_{1} \vDash \operatorname{some} \neg a f \operatorname{ter}(r c) \stackrel{(S)}{\mapsto} \operatorname{some} \neg a f t e r(r c):\left(s_{1} \models \neg a f t e r(r c) \rightarrow s_{2} \vDash \neg a f t e r(r c) \wedge \operatorname{sink}\right) \\
& s_{2} \models \neg a f t e r(r c) \wedge \operatorname{sink} \stackrel{(C)}{\mapsto}\left(s_{2} \models \neg a f t e r(r c) \& s_{2} \vDash \sin k\right)
\end{aligned}
$$

For convinience, we represent in the sequel the assertions like $s \vDash T$ appearing in the explanations only by $s$ (in the same way as we forget these assertions appearing in terms like $s \vDash T \& x)$. More, it is often desirable that action names also appear in explanations so as to establish a more direct correspondence with the program, as action names appear in the program, but not the states. Using these conventions, the explanation obtained in the preceding example is the following (rule $(X)$ ):

$$
\begin{aligned}
& \operatorname{pot}(a f t e r(t m) \wedge \text { some } \neg a f t e r(r c)):\left(s _ { 0 } \stackrel { e m } { \rightarrow } \left(s_{1} \vDash \text { after }(\text { em }) \text { \&some } \neg a f t e r(r c):\right.\right. \\
& \left.\left.\left(s_{1} \models \neg a f t e r(r c) \stackrel{\text { lost }}{\rightarrow}\left(s_{2} \models \neg a f t e r(r c) \& s_{2} \vDash \sin k\right)\right)\right)\right)
\end{aligned}
$$

- Properties of the system of rules.

- Termination : any complete explanation of an assertion $s \models f$ is finite.

- Completeness : if $s \vDash f$ then $X(s \models f) \neq \emptyset$

- Soundness : if $s \not f$ then $X(s \vDash f)=\emptyset$

\section{- Minimal explanations}

Consider the following restrictions of the rules $(P)$ and $(S)$, where the explanations of $\operatorname{pot}\left[f_{1}\right] f_{2}$ and some $\left[f_{1}\right] f_{2}$ are built with minimal $e s$ :

\begin{tabular}{|l|l|l|l|}
\hline$w \in W^{0}\left(s \in \operatorname{pot}\left[f_{1}\right] f_{2}\right)$ & $\operatorname{pot}\left[f_{1}\right] f_{2}: e\left(w, \operatorname{pot}\left[f_{1}\right] f_{2}\right)$ & $s=\operatorname{pot}\left[f_{1}\right] f_{2}$ & $\left(P^{0}\right)$ \\
\hline$w \in W^{0}\left(s \in \operatorname{some}\left[f_{1}\right] f_{2}\right)$ & some $\left[f_{1}\right] f_{2}: e\left(w, \operatorname{some}\left[f_{1}\right] f_{2}\right)$ & $s=\operatorname{some}\left[f_{1}\right] f_{2}$ & $\left(S^{0}\right)$ \\
\hline
\end{tabular}

The explanations obtained by applying only the rules $(I),(D),(C),\left(P^{0}\right),\left(S^{0}\right)$ and $(X)$ are called minimal. Because of the properties of minimal explicative sequences, only a 
finite number of rules $\left(P^{0}\right)$ (resp. $\left(S^{0}\right)$ ) can be applied to an assertion $s / p o t\left[f_{1}\right] f_{2}$ (resp. $\left.s=\operatorname{some}\left[f_{1}\right] f_{2}\right)$. This system of rules has the same properties as the general one, in particular, it is complete : every assertion has a minimal explanation.

\section{Simplification of the explanations}

As the programs into considerations and therefore the explanations may be large, it is important to be able to compress the explanations. We provide the user with the possibility to define an observation criterion by defining a sub-set $V$ of visible actions of Act, which are the only to appear in the explanations, and we associate the label $\lambda$ with the transitions without visible action. In the $e s$ of $s \models f$, there can be states $s_{1}, s_{2}$, which cannot be distinguished with respect to $f$ and $V$ in the following sense : they satisfy the same sub-formulas of $f$, and $s_{2}$ is reached from $s_{1}$ by a $\lambda$-path. A transition between two such states is a silent transition. In simplified execution sequences every maximal sequence of states related by silent transitions is replaced by exactly one of these states. The simplified es do not contain silent transitions. There are two possible methods to obtain simplified explanations. The first method is the one described above consisting of computing first an explanation and then simplifying it. A second method consists of first simplifying the model, and then computing the explanations in this simplified model. This is possible if we find a reduction method allowing to find the same simplified explanations than by using the first method. In this section, we define an observation criterion for a model, the simplified explanations, and then an explanational equivalence on models which preserves the simplified explanations.

\subsection{Observation criterion}

$\phi(f)$ is the set of formulas which can appear in an explanation of $f$, defined by :

$$
\begin{gathered}
\text { if } f \text { is a basic formula, } \phi(f)=\{f\}, \quad \phi\left(\operatorname{pot}\left[f_{1}\right] f_{2}\right)=\left\{\operatorname{pot}\left[f_{1}\right] f_{2}\right\} \cup \phi\left(f_{1}\right) \cup \phi\left(f_{2}\right) \\
\phi\left(\text { some }\left[f_{1}\right] f_{2}\right)=\left\{\text { some }\left[f_{1}\right] f_{2}\right\} \cup \phi\left(f_{1}\right) \cup \phi\left(f_{2}\right) \cup \phi\left(\neg f_{1}\right) \cup\{\operatorname{sink}\} \\
\phi\left(f_{1} \wedge \ldots \wedge f_{n}\right)=\phi\left(f_{1}\right) \cup \ldots \cup \phi(f n), \quad \phi\left(f_{1} \vee \ldots \vee f_{n}\right)=\phi\left(f_{1}\right) \cup \ldots \cup \phi\left(f_{n}\right)
\end{gathered}
$$

An observation criterion is a pair $C=(V, f)$ where $V$ is a sub-set of Act, and $f$ a formula. $C$ specifies which informations the user is interested in, by defining :

- A labelling of the transitions restricted to the visible actions. Let $V_{\lambda}=V \cup\{\lambda\}$ and $\sim \subset S \times\left(V_{\lambda} \cup\{\tau\}\right) \times S$ be the transition relation such that :

$$
s_{1} \stackrel{\beta}{\sim} s_{2} \Leftrightarrow s_{1} \stackrel{\alpha}{\rightarrow} s_{2} \text { and } \begin{cases}\text { if } \alpha \notin V \text { and } \phi\left(s_{1}\right)=\phi\left(s_{2}\right) & \text { then } \beta=\tau \\ \text { if } \alpha \notin V \text { and } \phi\left(s_{1}\right) \neq \phi\left(s_{2}\right) & \text { then } \beta=\lambda \\ \text { if } \alpha \in V & \text { then } \beta=\alpha\end{cases}
$$

$\tau$-transitions are the above defined silent transitions.

- A labelling of the states, restricted to the elements of $\mathcal{P}_{f}=\mathcal{P} \cap \phi(f)$.

By labelling the transitions and the states of $M$ according to $C$, we define a new model $M_{C}=\left(S, \sim, s_{0}, V_{\lambda} \cup\{\tau\}, \mathcal{P}_{f}, \Pi\right)$. In this model, the satisfaction relation for the sub-formulas 
of $f$ is the same : $\forall g \in \phi(f) . M, s \models g$ iff $M_{C}, s \models g$. Thus, the explanations of $s \models f$ in the model $M$ and in the model $M_{C}$ are the same, up to the labelling of the transitions.

\subsection{Simplification of the explanations}

Simplifying an explanation means generating a more compact form of it without losing any useful information. We define more formally the notion of simplified explanations defined above. The transformations concern the sequences of explanations, sub-terms of the explanation to simplify, where the corresponding es contains a $\tau$-path. There are three transformations depending on the form of the sequences :

\begin{tabular}{|c|c|}
\hline Initial sequence of explanations & Simplified sequence \\
\hline (1) $\ldots x_{1} \stackrel{\tau}{\sim} \ldots \stackrel{\sim}{\sim} x_{n-1} \stackrel{\alpha}{\sim} x_{n}$ where $\forall i<n . x_{i} \in X\left(s_{i}=g\right)$ & $\ldots x_{1} \stackrel{a}{\sim} x_{n}$ \\
\hline (2) $\ldots x_{1} \stackrel{\tau}{\sim} \ldots x_{k}\left(\stackrel{\tau}{\sim} \ldots \stackrel{\tau}{\sim} x_{n}\right)^{\omega}$ where $\forall i . x_{i} \in X\left(s_{i} \models g\right)$ & $\ldots\left(x_{1} \& \Delta\left(s_{1}\right)\right)$ \\
\hline (3) $\begin{aligned} \ldots x_{1} \stackrel{\mathcal{\tau}}{\sim} & \ldots \stackrel{\tau}{\sim} x_{n-1} \stackrel{\tau}{\sim} x_{n} \\
& \text { where } x_{i} \in X\left(s_{i} \vDash g_{1}\right)_{i<n} \text { and } x_{n} \in X\left(s_{n} \vDash g_{2}\right)\end{aligned}$ & $\begin{array}{c}\cdots x_{1}^{\prime} \\
\text { where } x_{1}^{\prime} \in X\left(s_{1} \models g_{2}\right)\end{array}$ \\
\hline
\end{tabular}

(1) It is not necessary to explain the satisfaction of $g$ for each of the $s_{i}$ on a $\tau$-path. The sequence $x_{1} \ldots x_{n-1}$ will be reduced to only one of these explanations, say the first one.

(2) We say that a state $s_{1}$ diverges, denoted by $\Delta\left(s_{1}\right)$, if it is possible to reach from $s_{1}$ a cycle of silent transitions, without executing any visible transition. On the $\tau$-path $s_{1} \ldots s_{k}\left(\ldots s_{n}\right)^{\omega}$, the $s_{i}$ are not distinguished, but we do not want to lose the information of the existence of an infinite sequence. The sequence of explanations is reduced to $x_{1}$, supplied with the fact that $s_{1}$ diverges, denoted by $\left(x_{1} \& \Delta\left(s_{1}\right)\right)$.

(3) This case happens for explanations of an assertion $s / p$ pot $\left[g_{1}\right] g_{2}$; the transitions $\left(s_{i}, s_{i+1}\right)$ are silent, thus, if $s_{n} \vDash g_{2}, \forall i . s_{i} \models g_{2}$ (the corresponding es is not minimal). Then, there exists $x_{1}^{\prime} \in w\left(s_{1} \models g_{2}\right)$ with which the sequence $x_{1} \ldots x_{n}$ can be identified (see [Ras90]).

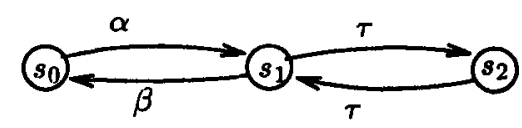

figure 3.

Example (figure 2). Let $f=$ pot some $\neg$ after $(\beta)$, the explanation of $s_{0}=f$ :

$f:\left(s_{0} \stackrel{\alpha}{\sim} s_{1} \stackrel{\tau}{\sim} \operatorname{some} \neg a f \operatorname{ter}(\beta):\left(s_{2} \models \neg a f \operatorname{ter}(\beta) \stackrel{\tau}{\sim} s_{1} \vDash \neg a f t e r(\beta)\right.\right.$

$$
\left.\left.\left(\stackrel{\tau}{\sim} s_{2} \vDash \neg a f \operatorname{ter}(\beta) \stackrel{\tau}{\sim} s_{1} \vDash \neg a f t e r(\beta)\right)^{\omega}\right)\right)
$$

can be simplified to : $f:\left(s_{0} \stackrel{a}{\sim} \operatorname{some} \neg a f \operatorname{ter}(\beta):\left(s_{1} \models \neg a f t e r(\beta) \& \Delta\left(s_{1}\right)\right)\right)$

\subsection{Explanational equivalence}

We define an equivalence between models which satisfies the following conditions :

1) it preserves the useful properties for the simplified explanations of $f:$ the satisfaction of the formulas appearing in an explanation of $f$, the sequences of visible actions on the es, 
and the properties of divergence.

2) it is done as weak as possible in order to allow efficient simplifications.

Definition 2 The explanational equivalence for $f, \approx_{f}$, is the weakest binary relation on $S$ such that:

$$
\begin{aligned}
s \approx_{f} s^{\prime} \Leftrightarrow & \phi(s)=\phi\left(s^{\prime}\right) \\
& \Delta(s) \Leftrightarrow \Delta\left(s^{\prime}\right) \\
& \forall \alpha \in V_{\lambda}:\left[\forall s_{1}:\left(s \stackrel{\tau * \alpha}{\sim} s_{1}\right) \Rightarrow\left(\exists s_{1}^{\prime} \mid s^{\prime} \stackrel{\tau * \alpha}{\sim} s^{\prime} 1 \text { and } s_{1} \approx_{f} s_{1}^{\prime}\right)\right] \\
& \text { and } \quad\left[\forall s_{1}^{\prime}:\left(s^{\prime} \stackrel{\tau * \alpha}{\sim} s_{1}^{\prime}\right) \Rightarrow\left(\exists s_{1}: s \stackrel{\tau * \alpha}{\sim} s_{1} \text { and } s_{1} \approx_{f} s_{1}^{\prime}\right)\right]
\end{aligned}
$$

Let $\leftrightarrow$ be the weakest congruence between explanations satisfying the rules :

$$
\begin{gathered}
\forall s_{1}, s_{2}: s_{1} \models f \leftrightarrow s_{2} \models f, \quad \Delta\left(s_{1}\right) \leftrightarrow \Delta\left(s_{2}\right) \\
\text { if } x_{1} \leftrightarrow x \text { and } x_{2} \leftrightarrow x, \text { then } x_{1} \& x_{2} \leftrightarrow x, \quad \text { if } x_{1} x_{2} \leftrightarrow x, \text { then }\left(x_{1} x_{2}\right)^{\omega} \leftrightarrow(x)^{\omega}
\end{gathered}
$$

The following property of the explanational equivalence has been proven in [Ras90] :

Proposition 4 If $s \approx_{f} s^{\prime}$, then for every simplified explanation $x$ of $s \neq f$, there exists a simplified explanation $x^{\prime}$ of $s^{\prime} \models f$ such that $x \leftrightarrow x^{\prime}$

Consequently, simplified explanations can be computed in any model of an equivalence class. The transition relation of the normal form of the model (the smallest equivalent model) is the $\tau$-closure of the transition relation, a silent loop beeing added for each diverging state. In computing such a model, the first step is $O\left(n_{\tau}^{3}\right)$, where $n_{\tau}$ is the number of silent transitions, the second one is of order of the size of the reduced model.

\section{Automatic generation of explanation}

We present hereafter an example of an explanation generation by CLÉo, which implements the method described in section 2. This tool is integrated in a verification tool in order to help the user in case of non validity of the specifications. An explanation of an assertion is built step by step, each step being the application of a rewrite rule ( $\$ 2.3$ ). After one step, the user selects an assertion in the partial explanation obtained, and get an explanation of this assertion. We consider in this example a program describing a token ring protocol.

\section{- Short description of the Chang-Roberts algorithm [CR79].}

The goal of this protocol is to realize a resource sharing with mutual exclusion between a set of stations $S_{i(i=1 \ldots n)}$ connected trough a virtual ring. A token moves along the ring. If $S_{i}$ wants to have acces to the resource, it waits for the token and transmits it to the next station after it has released the resource, The transmission lines are not reliable, thus the token may be lost. In this case, the stations elect one of the station who produces a new token. The only visible action at the higher level are the acces $\left(o p e n_{i}\right)$ and the release $\left(\right.$ close $\left._{i}\right)$ of the resource from the station $S_{i}$. The mutual exclusion property is expressed by the formulas :

$\operatorname{after}\left(\right.$ open $\left._{i}\right) \Rightarrow \neg \operatorname{pot}\left[\neg a f t e r\left(\left(\right.\right.\right.$ close $\left.\left._{i}\right)\right] a f t e r\left(\right.$ open $\left._{j}\right)$, for every pair $\left(S_{i}, S_{j}\right)$ of stations.

- Verification and diagnostics 
This protocol has been described in Lotos. It is known that the Chang-Roberts algorithm is correct, but our description in LOTOS was not. By using the CASAR system [GS90], it was possible to generate a graph for a fixed number of stations. For a ring of two stations, the

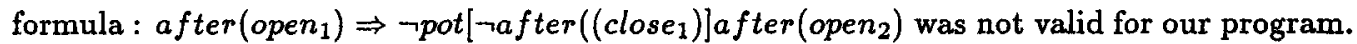
The verification tool gives us the list of the states (coded as integers) which do not satisfy it, i.e. who satisfy its negation $f=\operatorname{after}\left(\right.$ open $\left._{1}\right) \wedge \operatorname{pot}\left[\neg\right.$ after $\left(\right.$ close $\left.\left._{1}\right)\right]$ after $\left(\right.$ open $\left._{2}\right)$. Among this list, the user chooses a state $s$, for example $s=369$, in order to obtain an explanation of $s \equiv f$. First, CLÉo computes a sequence from the initial state $\left(s_{0}=1\right)$ to the chosen state, and applies of one rewrite rule to the assertion $s \neq f$ :

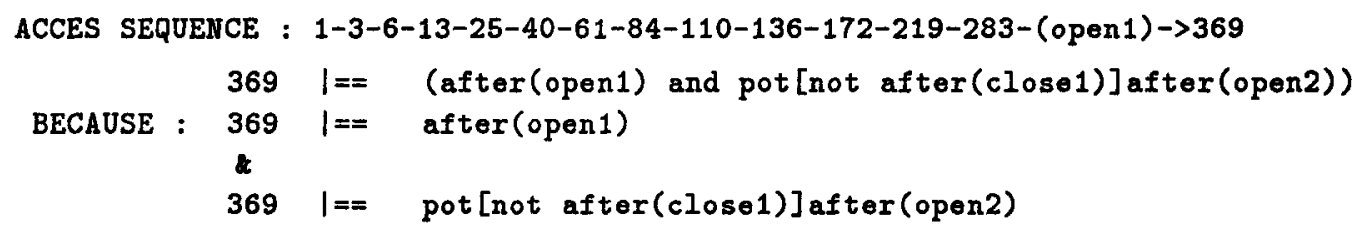

After that, the user selects an assertion in this partial explanation, and CLÉo computes the corresponding rewrite rule :

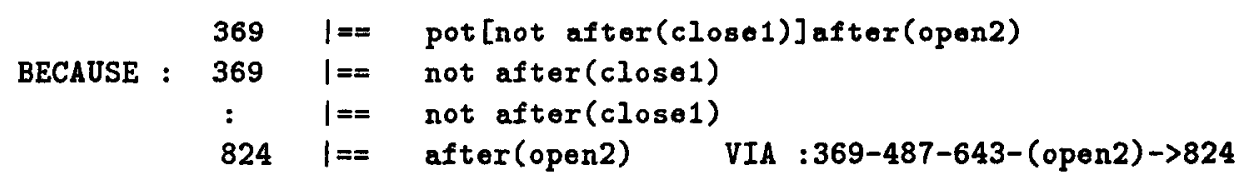

This explanation is build with the es 369.487 .643 .842 of $369 \models \operatorname{pot}\left[\neg a f t e r\left(\operatorname{close}_{1}\right)\right]$ after $\left(\right.$ open $\left._{2}\right)$. The acces sequence followed by the es forms an execution sequence where one can observe the sequence of actions : open open $_{2}$ whithout seeing the action close $_{1}$. Then, the mutual exclusion is not satisfied. Cassar allows to get the meaning of the silent transitions (the internal actions of the network) in terms of the source program. With the help of the explanation and of these actions, we could understand the cause of the error in the election process : it was possible to generate two tokens in the ring, and correct it.

\section{Conclusion}

We propose an error diagnosis method for specifications expressed in a branching time logic. The results are given for a particular logic, but our approach can be generalized to other specification formalisms.

In the set of the explanations, we have pointed out a finite subset of minimal explanations, sufficient to show all the reasons of non-satisfaction of $f$. The cost of computing an explanation of $s \neq f$ is the cost of a derivation of this assertion in the rewrite system. The generation of a minimal explicative sequence is the search of a shortest path in the graph of the program, and possibly for strongly connected components.

The explanations can be given in a simplified form depending on of visible actions defined 
by the user. For this aim, we have defined the explanational equivalence, such that the set of the simplified explanations of $f$ is the same in equivalent models. This equivalence relation depends on the formula, in order to be enough weak to allow efficient reductions.

An interesting application of our diagnosis method could be the generation of test sequences directed by the properties. In fact, the two problems are very close. Testing an implantation amounts to verifying that it has the same properties as its abstract model. By extracting from the model a finite set of sequences such that the property to verify is true because of them, one defines a set of test sequences for the property.

\section{References}

[AL88] M. Abadi and L. Lamport. The existence of refinement mappings. SRC 29, Digital Equipment Corporation, August 1988.

[Arn89] A. Arnold. MEC : a System for Constructing and Analysing Transition Systems. In Proceedings Workshop on Automatic Verification Methods for Finite State Systems, Grenoble, France, 1989.

[CES83] E. Clarke, E. A. Emerson, and A. P. Sistla. Automatic Verification of Finite State Concurrent Systems using Temporal Logic. In 10th Annual Symposium on Principles of Programming Languages, ACM, 1983.

[CR79] Ernest Chang and Rosemary Roberts. An Improved Algorithm for Decentralized Extrema-Finding in Circular Configurations of Processes. Communications of the $A C M, 22(5): 281-283$, may 1979 .

[EC82] E.A. Emerson and E.M. Clarke. Using branching time logic to synthtize synchronization skeletons. In Sci. Comput. Programming. Volume 2, 1982.

[GS90] Hubert Garavel and Joseph Sifakis. Compilation and Verification of LOTOS Specifications. In L. Logrippo, R. L. Probert, and H. Ural, editors, Proceedings of the 10th International Symposium on Protocol Specification, Testing and Verification (Ottawa, Canada), IFIP, North-Holland, Amsterdam, June 1990.

[Pnu86] A. Pnueli. Specification and Development in Reactive Systems. In Conf IFIP 86, North-Holland, 1986.

[Ras90] Anne Rasse. CLEO : diagnostic des erreurs en XESAR. Thèse de Doctorat, Institut National Polytechnique de Grenoble, June 1990.

[RRSV87] Jean-Luc Richier, Carlos Rodriguez, Joseph Sifakis, and Jacques Voiron. Verification in XESAR of the Sliding Window Protocol. In Harry Rudin and Colin H.

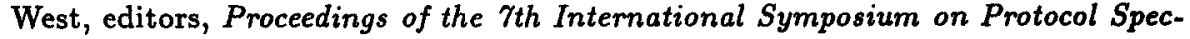
ification, Testing and Verification (Zurich), IFIP, North-Holland, May 1987. 